\title{
Effect of Probiotics on Metabolic Syndrome
}

\author{
Tomoyuki Kawada \\ Department of Hygiene and Public Health, Nippon Medical School, Tokyo, Japan
}

\section{Dear Editor,}

Dong et al. [1] conducted a meta-analysis of 18 clinical studies regarding the effect of probiotic foods or supplements with Lactobacillus and Bifidobacterium on components of metabolic syndrome (MetS). Although there were no significant differences of body mass index, body fat mass, waist circumference, hip circumference, waist-to-hip ratio, systolic blood pressure, diastolic blood pressure, fasting glucose, fasting insulin, total cholesterol, high-density lipoprotein cholesterol, hemoglobin A1c, or triglycerides between the intervention and control groups, there was a significant standardized mean difference in the body fat percentage and low-density lipoprotein cholesterol between the intervention and control groups. I have some concerns about their study with special reference to blood pressure and glucose metabolism.

First, Tenorio-Jiménez et al. [2] overviewed the effect of probiotics on the components of MetS in subjects without type 2 diabetes. Although they did not conduct a meta-analysis with 9 clinical studies, probiotics intake in patients with MetS resulted in improvements in body mass index, blood pressure, glucose metabolism, and lipid profile in some studies. They only cited the study outcomes of each paper, and conclusive remarks cannot be determined. They cited a meta-analysis by Dong et al. [1], and mentioned the study limitation regarding heterogeneities. But scientific evidence might be derived from a meta-analysis of randomized clinical trials. Dong et al. [1] presented heterogeneities on each component of MetS, and handled specific probiotics. I am afraid that selected papers for reviews were almost different and selection criteria might be important for conducting a meta-analysis.

Second, Khalesi et al. [3] conducted a meta-analysis of 9 clinical studies regarding the effect of probiotics on blood pressure. Probiotic consumptions significantly changed systolic blood pressure by $-3.56 \mathrm{~mm} \mathrm{Hg}$ (95\% CI -6.46 to -0.66$)$ and diastolic blood pressure by $-2.38 \mathrm{~mm} \mathrm{Hg}$ (95\% CI -2.38 to -0.93 ) compared with control groups. As there were significant heterogeneities and the results differed in different contents of probiotics. This paper was not cited by Dong et al. [1], and the published year of each paper differed. A meta-analysis was conducted by using almost different papers, and the advantage of probiotics for controlling blood pressure should be specified by further studies.

Finally, Dong et al. [1] recognized that there were no significant standardized mean differences in the fasting glucose and fasting insulin between the intervention and control groups. There were no heterogeneities regarding fasting glucose and fasting insulin, and I recommend the authors specifying the effect of probiotics on insulin resistance such as homeostasis model assessment of insulin resistance.

\section{Acknowledgement}

I express my appreciation to Dr. Toshiaki Otsuka for his comment on my manuscript.

\section{Disclosure Statement}

The author has no conflicts of interest to declare.

\section{Funding Sources}

There are no funding sources to declare.

\section{Author Contributions}

T.K. wrote a draft and revised it.

\section{karger@karger.com www.karger.com/anm \\ ๑) 2020 S. Karger AG, Basel}

Karger
Tomoyuki Kawada

Department of Hygiene and Public Health

Nippon Medical School, 1-1-5 Sendagi

Bunkyo, Tokyo 113-8602 (Japan)

E-Mail kawada@nms.ac.jp 


\section{References}

1 Dong Y, Xu M, Chen L, Bhochhibhoya A. Probiotic foods and supplements interventions for metabolic syndromes: A systematic review and meta-analysis of recent clinical trials. Ann Nutr Metab. 2019;74(3):224-41.
2 Tenorio-Jiménez C, Martínez-Ramírez MJ, Gil Á, Gómez-Llorente C. Effects of probiotics on metabolic syndrome: A systematic review of randomized clinical trials. Nutrients. 2020;12(1):E124.
3 Khalesi S, Sun J, Buys N, Jayasinghe R. Effect of probiotics on blood pressure: a systematic review and meta-analysis of randomized, controlled trials. Hypertension. 2014;64(4): 897-903. 